\title{
Nicotinamide Phosphoribosyltransferase
}

National Cancer Institute

\section{Source}

National Cancer Institute. Nicotinamide Phosphoribosyltransferase. NCI Thesaurus.

Code C30138.

Nicotinamide phosphoribosyltransferase (491 aa, $~ 56 \mathrm{kDa}$ ) is encoded by the human

NAMPT gene. This protein plays a role in both NAD biosynthesis and

immunomodulation. 